\title{
Traitement du cancer par thérapie cellulaire : cellules souches embryonnaires versus cellules souches adultes. Quels sont les aspects éthiques ?
}

\author{
Cancer treatment based on cellular therapy: cells versus adult stem cells. \\ What are the ethicals issues?
}

\author{
D. Sawadogo \\ C) Springer-Verlag France 2010
}

En 1981, Evans et Kaufman [1] ont isolé et cultivé des cellules souches pluripotentes embryonnaires de souris, ce qui lui a valu le prix Nobel de médecine en physiologie en 2007. Thomson et al. [2], 17 ans plus tard, en 1998, ont identifié et cultivé les cellules souches pluripotentes issues de blastocytes humains. Il initie ainsi ce qu'on appelle « la décennie prodigieuse des cellules souches » qui va de 1998 à 2008 [3].

La thérapie cellulaire repose sur le transfert in vivo de cellules ou de tissus produits in vitro pour le traitement de tissus ou d'organes in situ. La thérapie cellulaire est l'un des piliers de la médecine régénérative qui représente l'un des grands espoirs du futur. Il s'agit d'une médecine personnalisée. Il est vrai que d'un point de vue clinique, ce serait un progrès remarquable que de pouvoir disposer de n'importe quel type de culture de cellules, de tissus ou d'organes pour traiter les maladies. Cette médecine régénérative présenterait de nombreux avantages dont l'un des plus importants serait la réparation de tissus abîmés dans certaines affections telles que la maladie de Parkinson ou d'Alzheimer, l'infarctus du myocarde et le diabète. Bien avant les maladies dégénératives, c'est dans les affections cancéreuses que la médecine régénérative a acquis ses lettres de noblesse. En effet, l'intensification thérapeutique avec greffe de cellules souches hématopoiétiques est un outil incontournable dans le traitement des leucémies, du myélome multiple, des lymphomes et de bien d'autres cancers tels que les cancers du sein, les glioblastomes, etc. Après leur extraction, leur manipulation et leur administration, les cellules souches hématopoïétiques permettent de régénérer les moelles osseuses qui auraient été envahies par les cellules cancéreuses ou mises en aplasies

D. Sawadogo $(\bowtie)$

Département d'hématologie, immunologie, biologie cellulaire, unité de formation et de recherche (UFR) des sciences pharmaceutiques et biologiques, université de Cocody, 08 BP 2308 Abidjan 08, Côte-d'Ivoire

e-mail : dunisawadogo@yahoo.fr chimio- ou radio-induites. C'est ainsi que l'on peut guérir ou sauver la vie de nombreux patients.

La thérapie cellulaire dans les affections cancéreuses repose sur l'utilisation de cellules souches adultes qui proviennent de la moelle osseuse, du sang de cordon ombilical des nouveau-nés ou du sang des adultes [4].

La thérapie cellulaire déclencha dès le début un problème très délicat lié à l'origine des cellules souches utilisées : cellules souches adultes ou cellules souches embryonnaires [3]. Il y avait deux alternatives avec deux camps. Il y avait le groupe de ceux qui pensaient que les cellules souches adultes avaient la capacité de réparer les tissus lésés [5-7]. D’autres chercheurs, par contre, estimaient que les véritables cellules souches étaient les cellules embryonnaires [1,8]. Les cellules souches adultes ne posséderaient pas la même capacité de différenciation et de prolifération que les cellules souches embryonnaires.

Les cellules souches embryonnaires sont des cellules souches pluripotentes qui sont issues de la différenciation des cellules souches totipotentes. Elles proviennent des embryons qui n'ont pas été utilisés lors de la fécondation in vitro, des embryons ayant été créés dans le but d'être une source de cellules souches, des embryons obtenus par clonage des restes de fœtus après les avortements. Elles possèdent diverses caractéristiques dont une des plus importantes est d'avoir un potentiel régénératif très important. Elles peuvent se différencier dans tous les tissus de l'organisme. Elles ont aussi le pouvoir de se transformer en cellules tumorales, qui sont des cellules instables. Les cellules souches embryonnaires présenteraient de fausses promesses concernant leur potentiel thérapeutique [3].

Les cellules souches adultes ont été identifiées dans plusieurs tissus : moelle osseuse, cerveau, sang périphérique, système digestif, pancréas, sang de cordon ombilical, vaisseaux sanguins. Il s'agit de cellules souches multipotentes issues de la différenciation des cellules souches pluripotentes. Elles sont capables de donner naissance à différentes lignées cellulaires d'un tissu donné. Elles sont la base du 
renouvellement naturel d'un tissu et de sa réparation à la suite d'une lésion. Elles sont déjà utilisées dans des traitements de plus d'une centaine de maladies. Leur principal avantage provient du fait qu'elles peuvent être obtenues sans porter préjudice ou nuire à l'intégrité physique du donneur. Elles ne sont pas aussi nombreuses que les cellules souches embryonnaires, elles ne se reproduisent pas aussi facilement et sont plus difficiles à rencontrer, car elles sont dispersées au milieu de tissus et de cellules bien différenciées. Leur inconvénient majeur serait d'être plus différenciées que les cellules souches embryonnaires, et donc d'avoir un potentiel régénératif plus restreint que les cellules souches embryonnaires [3]. Cette affirmation a été battue en brèche par les travaux de plusieurs auteurs dont Jiang et al. [5-7]. Cette équipe a prouvé l'existence de cellules souches pluripotentes dans la moelle osseuse de l'adulte. Il s'agit des mesenchymal adult pluripotent stem cell. Ces cellules ont les mêmes caractéristiques que les cellules souches embryonnaires.

Il existe aussi des cellules souches pluripotentes adultes induites ou induced pluripotent stem cells qui ont été mises au point par le groupe de Shinya Yamanaka de l'université de Kyoto, Japon [9] par la technique de « reprogrammation » nucléaire en utilisant des vecteurs viraux. Ils ont réussi à transformer des cellules somatiques différenciées en cellules souches pluripotentes.

Jiang et al. [7] et Takahashi et al. [9] ont ainsi montré par leurs découvertes que le principal inconvénient de l'utilisation des cellules souches adultes pouvait être surmonté. Les cellules souches pluripotentes peuvent être aussi retrouvées chez des adultes.

Il ne faut pas oublier que selon Lacadena [3], qui a fait dans son article toute une révision des principales découvertes concernant les cellules souches de 1998 à 2008, les cellules souches embryonnaires et adultes sont deux réalités biologiques différentes avec des fonctions qui sont différentes. Les premières, embryonnaires, servent à la génération d'un être humain. Les secondes ont pour rôle de réparer et de régénérer les dommages dans les tissus et les organes.

D'un point de vue éthique, l'utilisation de cellules souches embryonnaires et de cellules souches adultes n'a pas les mêmes implications. L'utilisation de cellules souches adultes ne comporte aucune réserve éthique, sauf celle générale liée aux conditions qu'il faut toujours respecter dans la recherche. L'utilisation des cellules souches embryonnaires par contre implique la destruction de l'embryon. Un embryon humain n'est pas un tas de cellules, c'est un être humain. Il est doté d'une unité génétique, il est unique. Il existe une continuité biologique entre l'embryon, le fotus, l'enfant et l'adulte qu'il va devenir. Il possède une autonomie dans son développement. Il a la capacité de développer, de contrôler, de coordonner les différentes étapes de son processus de formation [10]. Même si les embryons n'ont pas la possibilité de vivre, ils ne peuvent tout simplement pas être utilisés comme « matériel biologique » à usage thérapeutique.

Traitement du cancer par intensification thérapeutique associée à une thérapie cellulaire : cellules souches embryonnaires versus cellules souches adultes. De quel côté la balance va-t-elle pencher pour les Africains?

Le fardeau du cancer est inégalement réparti entre les pays à travers le monde. Le niveau de développement socioéconomique influe sur les facteurs de risque, la mortalité du cancer. L'Afrique est l'un des continents les plus touchés.

Les perspectives épidémiologiques des cancers qui se profilent d'ici à l'horizon de 2020 prévoient une progression continue en termes d'incidence et de mortalité. Si cet impact des pathologies tumorales est individuellement et collectivement à déplorer à l'échelle mondiale, il a davantage de conséquences néfastes dans les pays en développement sur lesquels pèseront $72 \%$ du fardeau global de la maladie [11]. Chaque année plus de dix millions de nouveaux cas sont reportés, dont six millions dans les pays aux ressources limitées, représentant ainsi la moitié des cas mondiaux ; quatre millions en mourront, soit un million de plus que ceux décédés du VIH/sida [12]. Cette progressive incidence des cancers, dont les hémopathies malignes, serait probablement due à une plus grande exposition des personnes aux facteurs carcinogènes, liée à la modernisation du mode de vie. Elle pourrait aussi correspondre à une augmentation relative des cas en rapport avec l'amélioration des moyens d'investigations diagnostiques. En effet, à Abidjan, l'incidence hospitalière des hémopathies malignes n'a cessé de croître. Elle est passée de 43,2 (1972 à 1981) à 53,5 (1991 à 2003) et à 168,1 (1995 à 2004) [13].

En Côte-d'Ivoire par exemple, comme dans de nombreux pays africains, le traitement des hémopathies malignes repose essentiellement sur la chimiothérapie, parce que nous ne disposons pas de la radiothérapie. La réponse thérapeutique se caractérise par un faible taux de rémission complète, un nombre élevé de décès.

La lutte contre le cancer en Afrique au sud du Sahara est confrontée à de nombreux défis, et pourtant des interventions à portée des pays à revenus faibles existent. La thérapie cellulaire en fait partie. L'intensification thérapeutique myéloablative ou non avec la greffe de cellules souches adultes hématopoiétiques de sang de cordon ombilical pourrait améliorer très nettement le pronostic des cancers, notamment des hémopathies malignes [4].

Les fonds concernant la recherche viennent presque toujours des pays du Nord. Il serait ingénu de sous-estimer l'influence des lobbies en faveur de la recherche sur les embryons et le clonage humain. Ces groupes de pression défendent les intérêts économiques des entreprises biotechnologiques et des cliniques privées de fécondations in vitro liées à des centres de recherche sur les embryons [14]. 
Traitement du cancer par intensification thérapeutique associée à une thérapie cellulaire : cellules souches embryonnaires versus cellules souches adultes. De quel côté la balance va-t-elle pencher pour les Africains ? Pour effectuer le choix juste, les chercheurs et tous les acteurs de la santé en Afrique ne devraient pas oublier les principes éthiques suivants : tout ce qui est techniquement possible n'est pas éthiquement concevable; la fin ne justifie pas les moyens ; l'homme n'est pas un moyen mais une fin en lui même.

\section{Références}

1. Evans MJ, Kaufman MH (1981) Establishment in culture of pluripotential cells from mouse embryos. Nature 292:154-6

2. Thomson JA, Itskovitz-Eldor J, Shapiro SS, et al (1998) Embryonic stem cells derived from human. Science 282:1145-7

3. Lacadena JR (2008) La década prodigiosa de las células troncales (1998-2008) y la medecina regeneraitva. Moralia 31:65-95

4. Sawadogo D, Inwoley KA, Sangaré M, Yayo Ayé M (2008) Caractérisation des cellules souches hématopoiétiques par les antigènes membranaires CD34, CD71 à Abidjan, Côte-d'Ivoire. J Sci Pharm Biol 1:57-62
5. Bjornson CRR, Rietze RI, Reynolds BA, et al (1999) Turning brain into blood: a hematopoietic fate adopted by adult neural stem cells in vivo. Science 283:534-7

6. Mezey E, Chandross KJ, Harta G, et al (2000) Turning blood into brain: cells bearing neuronal antigens generated in vivo from bone marrow. Science 290:1779-82

7. Jiang Y, Jahagirdar BN, Reinhardt RL, et al (2002) Pluripotency of mesenchymal stem cells derived from adult marrow. Nature 418:41-9

8. Chung Y, Klimanskaya I, Becker S, et al (2006) Embryonic and extraembryonic stem cells lines derived from single mouse blastomeres. Nature 439:216-9

9. Takahashi K, Tanabe K, Ohnuki M, et al (2007) Induction of pluripotent cells from adult human fibroblasts by defined factors: Cell 131:1-12

10. Andomo R (2004) La dimensión biológica de la personalidad humana: el debate sobre el estatuto del embrión. Cuaderno de Bioetica 53:29-36

11. Ly A (2009) Les défis de la progression des cancers en Afrique. JAC $1: 1-3$

12. Dangou JM, Sambo BH, Moeti M, Diarra-Nama AJ (2009) Prévention et lutte contre le cancer dans la région africaine de l'OMS : un appel à l'action 1:56-60

13. Sawadogo D (2009) Caractéristiques épidémiologiques des patients atteints d'hémopathies malignes à Abidjan autour de la décennie 1995-2004. JAC 1:4-11

14. Lopez Moratalla N (2005) Lobby of embryionic stem cells. Background of the cloning fraud. Cuaderno de Bioética 58: 419-31 